\title{
A Vietnamese Dataset for Evaluating Machine Reading Comprehension
}

\author{
Kiet Van Nguyen ${ }^{1,2}$, Duc-Vu Nguyen ${ }^{1,2}$, Anh Gia-Tuan Nguyen $^{1,2}$, Ngan Luu-Thuy Nguyen ${ }^{1,2}$ \\ ${ }^{1}$ University of Information Technology, Ho Chi Minh City, Vietnam \\ ${ }^{2}$ Vietnam National University, Ho Chi Minh City, Vietnam \\ \{kietnv, vund, anhngt, ngannlt\}@uit.edu.vn
}

\begin{abstract}
Over 97 million people speak Vietnamese as their native language in the world. However, there are few research studies on machine reading comprehension (MRC) for Vietnamese, the task of understanding a text and answering questions related to it. Due to the lack of benchmark datasets for Vietnamese, we present the Vietnamese Question Answering Dataset (UIT-ViQuAD), a new dataset for the low-resource language as Vietnamese to evaluate MRC models. This dataset comprises over 23,000 human-generated question-answer pairs based on 5,109 passages of 174 Vietnamese articles from Wikipedia. In particular, we propose a new process of dataset creation for Vietnamese MRC. Our in-depth analyses illustrate that our dataset requires abilities beyond simple reasoning like word matching and demands single-sentence and multiple-sentence inferences. Besides, we conduct experiments on state-of-the-art MRC methods for English and Chinese as the first experimental models on UIT-ViQuAD. We also estimate human performance on the dataset and compare it to the experimental results of powerful machine learning models. As a result, the substantial differences between human performance and the best model performance on the dataset indicate that improvements can be made on UIT-ViQuAD in future research. Our dataset is freely available on our website ${ }^{1}$ to encourage the research community to overcome challenges in Vietnamese MRC.
\end{abstract}

\section{Introduction}

Machine reading comprehension (MRC) is an understanding natural language task that requires computers to understand a text and then answer questions related to it. MRC is an essential core for a range of natural language processing applications such as search engines and intelligent agents (Alexa, Google Assistant, Siri, and Cortana) In order to evaluate MRC models, gold standard resources with questionanswer pairs based on documents have to be collected or created by human. Building a benchmark dataset plays a vital role in evaluating natural language processing models, especially for a low-resource language like Vietnamese.

Typical gold standard MRC resources for English are span-extraction MRC datasets (Rajpurkar et al., 2016; Rajpurkar et al., 2018; Trischler et al., 2017), cloze-style MRC datasets (Hermann et al., 2015; Hill et al., 2015; Cui et al., 2016), multiple-choice MRC datasets (Richardson et al., 2013; Lai et al., 2017) and conversation-based MRC datasets (Reddy et al., 2019; Sun et al., 2019). For other languages, there are the Chinese dataset of the span-extraction MRC (Cui et al., 2019b; Duan et al., 2019), the traditional Chinese dataset of MRC (Shao et al., 2018), the Chinese user-query-log-based dataset of DuReader ( $\mathrm{He}$ et al., 2018), and the Korean MRC dataset (Lim et al., 2019). Due to the rapid development of the reading comprehension datasets, various neural network-based models have been proposed and made a significant advancement in this research field such as Match-LSTM (Wang and Jiang, 2016), BiDAF (Seo et al., 2017), R-Net (Wang et al., 2017), DrQA (Chen et al., 2017), FusionNet (Huang et al., 2018), FastQA (Weissenborn et al., 2017), and QANet (Yu et al., 2018). Pre-trained language models, BERT

\footnotetext{
${ }^{1}$ https://sites.google.com/uit.edu.vn/uit-nlp/datasets-projects

This work is licensed under a Creative Commons Attribution 4.0 International License. License details: http://creativecommons.org/licenses/by/4.0/
} 
(Devlin et al., 2019) and XLM-R (Conneau et al., 2020) have recently become extremely popular and achieved state-of-the-art performances for MRC tasks.

Vietnamese is a language with few resources for natural language processing. The dataset for MRC introduced by (Nguyen et al., 2020) consists of 2,783 multiple-choice questions and answers based on a set of 417 Vietnamese texts which are used for evaluating the reading comprehension skill for $1^{\text {st }}$ to $5^{\text {th }}$ graders. However, this dataset is relatively small in size to evaluate deep learning models for the Vietnamese MRC. Thus, we aim to build a new large dataset for evaluating Vietnamese MRC.

Though the deep learning approach has surpassed the human performance on the SQuAD (Rajpurkar et al., 2016) and NewsQA (Trischler et al., 2017) datasets, we wonder if these state-of-the-art models could also achieve similar performances on datasets of different languages. To further enhance the development of the MRC, we build a span-extraction MRC dataset where answers to questions are always spans from a given text for Vietnamese. Figure 1 shows several examples for Vietnamese span-extraction reading comprehension. In this study, we have four main contributions described as follows.

- We create a benchmark dataset for evaluating Vietnamese MRC: UIT-ViQuAD comprises 23,074 human-generated question-answer pairs based on 5,109 passages of 174 Vietnamese Wikipedia articles. The dataset is available freely on our website ${ }^{2}$ for research purposes.

- To gain thorough insights into the dataset, we analyze the dataset according to different linguistic aspects including length-based analysis (question length, answer length, and passage length) and type-based analysis (question type, answer type, and reasoning type).

- To achieve first MRC evaluation on UIT-ViQuAD, we conduct experiments with MRC models which are state-of-the-art for English and Chinese. Then, we compare performances between the machine models and humans in terms of different linguistic aspects. These in-depth analyses provide insights into span-based MRC in Vietnamese.

- Cross-lingual MRC (Cui et al., 2019a) is a new trend in natural language processing. Our proposed MRC dataset for Vietnamese could also be a resource for cross-lingual study along with other similar datasets such as SQuAD, CMRC, and KorQuAD.

\footnotetext{
Passage: Nước biển có độ mặn không đồng đều trên toàn thế giới mặc dù phần lớn có độ mặn nằm trong khoảng từ $\overline{3,1 \%}$ tới 3,8\%. Khi sự pha trộn với nước ngọt đổ ra từ các con sông hay gần các sông băng đang tan chảy thì nước biển nhạt hơn một cách đáng kể. Nước biển nhạt nhất có tại vịnh Phần Lan, một phần của biển Baltic.

(English: Seawater has uneven salinity throughout the world although most salinity ranges from 3.1\% to $3.8 \%$.

When the mix with freshwater pouring from rivers or near glaciers is melting, the seawater is significantly lighter. The lightest seawater is found in the Gulf of Finland, a part of the Baltic Sea.)

Question: Độ mặn thấp nhất của nước biển là bao nhiêu? (English: What is the lowest salinity of seawater?)

Answer: 3.1\% (English: 3.1\%)

Question: Nước biển ở đâu có hàm lượng muối thấp nhất? (English: Where is the lowest salt content?)

Answer: Vịnh Phần Lan. (English: Gulf of Finland.)
}

Figure 1: Several examples for Vietnamese span-extraction reading comprehension. The English translations are also provided for comparison.

The rest of this paper is structured as follows. Section 2 reviews existing datasets. Section 3 introduces the creation process of our dataset. In-depth analyses of our dataset are presented in Section 4. Then Section 5 presents our experiments and analysis results. Finally, Section 6 presents conclusions and directions for future work.

\section{Existing datasets}

Because we aim to build a span-based MRC dataset for Vietnamese, a range of recent span-extraction MRC datasets such as SQuAD (Rajpurkar et al., 2016), NewsQA (Trischler et al., 2017), CMRC (Cui et

${ }^{2}$ https://sites.google.com/uit.edu.vn/uit-nlp/datasets-projects 
al., 2019b), and KorQuAD (Lim et al., 2019) is reviewed in this section. These datasets are described as follows.

SQuAD is one of the most popular English datasets of the span-based MRC. Rajpurkar et al. (2016). proposed SQuAD v1.1 created by crowd-workers on 536 Wikipedia articles with 107,785 questionanswer pairs. SQuAD v2.0 (Rajpurkar et al., 2018) was released with adding over 50,000 unanswerable questions created adversarially by crowd-workers according to the original ones.

NewsQA is another English dataset proposed by Trischler et al. (2017), consisting of 119,633 question-answer pairs generated by crowd-workers on 12,744 news articles from CNN news. This dataset is similar to $\mathrm{SQuAD}$ because the answer to each question is a text segment of arbitrary length in the corresponding news article.

CMRC (Cui et al., 2019b) is a span-extraction dataset for Chinese MRC introduced in the Second Evaluation Workshop on Chinese Machine Reading Comprehension 2018, comprising approximately 20,000 human-annotated questions on Wikipedia articles.

KorQuAD (Lim et al., 2019) is a Korean dataset for span-based MRC, consisting of over 70,000 human-generated question-answer pairs on Korean Wikipedia articles.

These datasets are studied in the development and evaluation of various deep neural network models in NLP, such as Match-LSTM (Wang and Jiang, 2016), BiDAF (Seo et al., 2017), R-Net (Wang et al., 2017), DrQA (Chen et al., 2017), FusionNet (Huang et al., 2018), FastQA (Weissenborn et al., 2017) and QANet (Yu et al., 2018). Most recently, BERT (Devlin et al., 2019) and XLM-R (Conneau et al., 2020), which are powerful models trained on multiple languages, have obtained state-of-the-art performances on MRC datasets.

Until now, there has not been any datasets of Vietnamese Wikipedia texts for span-based MRC research. As mentioned above, the datasets are benchmarks for the MRC task and may be used for organizing a challenge which encourages researchers to explore the best processing models. Therefore, this is our primary motivation to create the new dataset for Vietnamese MRC.

\section{Dataset creation}

In this section, we introduce our proposed process of MRC dataset creation for the Vietnamese language. In particular, we build our UIT-ViQuAD dataset through five phases consisting of worker recruitment, passage collection, question-answer sourcing, validation and additional answers collection. These phases are described in detail as follows.

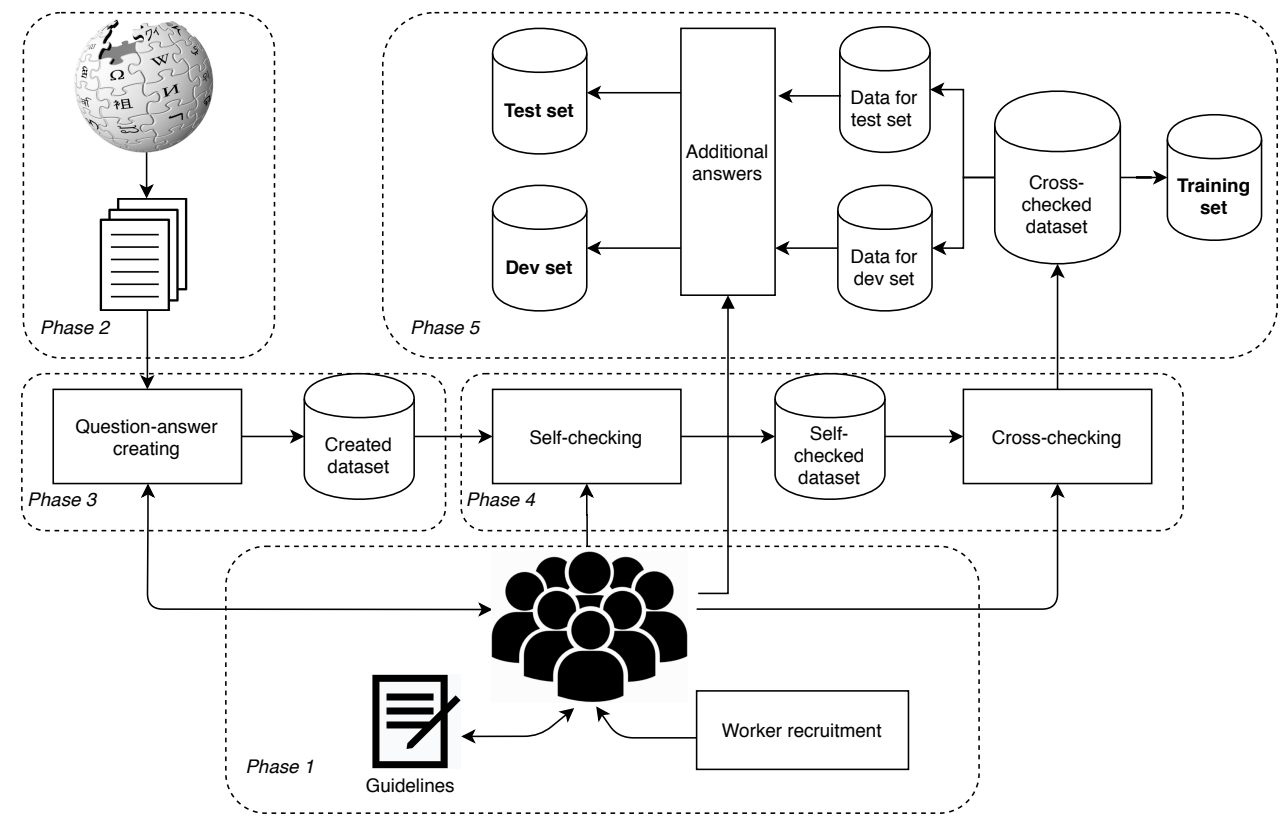

Figure 2: The overview process of creating our dataset UIT-ViQuAD. 
Phase 1 - Worker recruitment: The quality of a dataset depends on high-quality workers and the process of data creation. In this section, we present worker recruitment for creating our dataset according to a rigorous process, consisting of four different stages. (1) People apply to become workers for creating answer-question pairs of the dataset; (2) Selected people are excellent at general knowledge and passed our reading comprehension test; (3) Official workers are carefully trained over 500 question-answer pairs and cross-checked their created data to detect common mistakes that can be avoided when creating data.

Phase 2 - Passage collection: Similar to SQuAD, we also use Project Nayuki's Wikipedia's internal PageRanks $\unlhd^{3}$ to obtain a set of the top 5,000 Vietnamese articles, from which we choose randomly 151 articles for dataset creation. Each passage corresponds to a paragraph in an article. Images, figures, and tables are excluded. We also delete passages shorter than 300 characters or containing many special characters and symbols.

Phase 3 - Question-answer sourcing: Workers comprehend each passage and then create questions and corresponding answers. During the question and answer creation, workers follow rules which are: (1) Workers are required to create at least three questions per passage. (2) Workers are encouraged to ask questions in their own words. (3) Answers are text spans in the passage that are used to answer the questions. (4) Workers are encouraged to make diversities in questions, answers, and reasoning.

Phase 4 - Question and answer validation: In this phase, workers perform two different sub-phases to check mistakes in question-answer pairs including self-checking and cross-checking. The mistakes are classified into five different categories: unclear questions, misspellings, incorrect answers, lack or excess of information in answers, and incorrect-boundary answers. The two sub-phases are described as follows.

- Self-checking: Workers revise their question-answer pairs themselves.

- Cross-checking: Workers cross-check each other's question-answer pairs. If they discover any mistakes in the dataset, they discuss with each other to correct the mistakes.

Phase 5 - Additional answers collection: To evaluate the quality of dataset creation, for the development and test datasets, we add three more answers for each question by different workers in addition to the original answer. During this phase, the workers cannot see each other's answer and they are encouraged to make diversified answers.

\section{Dataset analysis}

\subsection{Overall statistics}

The statistics of the training (Train), development (Dev) and test (Test) sets of our dataset are described in Table 1. The number of questions of UIT-ViQuAD is 23,074. In the table, the numbers of articles and passages, the average lengths ${ }^{6}$ of questions and answers, and vocabulary sizes are also presented.

\begin{tabular}{lrrrr}
\hline & Train & Dev & Test & \multicolumn{1}{c}{ All } \\
\hline Number of articles & 138 & 18 & 18 & 174 \\
Number of passages & 4,101 & 515 & 493 & 5,109 \\
Number of questions & 18,579 & 2,285 & 2,210 & $\mathbf{2 3 , 0 7 4}$ \\
Average passage length & 153.9 & 147.9 & 155.0 & 153.4 \\
Average question length & 12.2 & 11.9 & 12.2 & 12.2 \\
Average answer length & 8.1 & 8.4 & 8.9 & 8.2 \\
Vocabulary size & 36,174 & 9,184 & 9,792 & 41,773 \\
\hline
\end{tabular}

Table 1: Overview statistics of the UIT-ViQuAD dataset.

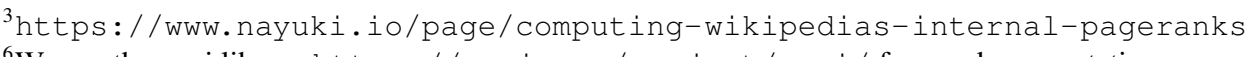

${ }^{6}$ We use the pyvi library https://pypi.org/project/pyvi/for word segmentation. 


\subsection{Length-based analysis}

We present statistics of our dataset according to three types of length including question length (see Table 2), answer length (see Table 2), and passage length (see Table 3). The 11-15-word questions of the dataset account for a high proportion of $45.29 \%$. The answers are mostly from 1 to 10 word lengths, accounting for $73.68 \%$. The length of passages is largely from 101 to 200 words with $73.13 \%$. These analyses show that our dataset has its own characteristics.

\begin{tabular}{crrrrrrrr}
\hline \multirow{2}{*}{ Length } & \multicolumn{4}{c}{ Question } & \multicolumn{4}{c}{ Answer } \\
\cline { 2 - 9 } & Train & \multicolumn{1}{c}{ Dev } & \multicolumn{1}{c}{ Test } & \multicolumn{1}{c}{ All } & Train & Dev & \multicolumn{1}{c}{ Test } & \multicolumn{1}{c}{ All } \\
\hline $1-5$ & 1.03 & 1.44 & 0.95 & 1.06 & $\mathbf{5 4 . 1 2}$ & $\mathbf{5 0 . 6 3}$ & $\mathbf{5 2 . 2 6}$ & $\mathbf{5 3 . 6 0}$ \\
$6-10$ & 35.99 & 38.38 & 33.21 & 35.96 & 19.95 & 22.14 & 19.10 & 20.08 \\
$11-15$ & $\mathbf{4 4 . 9 7}$ & $\mathbf{4 4 . 2 9}$ & $\mathbf{4 9 . 0 5}$ & $\mathbf{4 5 . 2 9}$ & 10.86 & 10.81 & 10.81 & 10.85 \\
$16-20$ & 15.01 & 13.61 & 14.07 & 14.78 & 6.28 & 7.48 & 6.83 & 6.45 \\
$>20$ & 3.00 & 2.28 & 2.71 & 2.90 & 8.80 & 8.93 & 11.00 & 9.02 \\
\hline
\end{tabular}

\begin{tabular}{crrrr}
\hline \multirow{2}{*}{ Length } & \multicolumn{4}{c}{ Passage } \\
\cline { 2 - 5 } & Train & \multicolumn{1}{c}{ Dev } & \multicolumn{1}{c}{ Test } & \multicolumn{1}{c}{ All } \\
\hline$<101$ & 11.41 & 10.10 & 11.16 & 11.25 \\
$101-150$ & $\mathbf{4 7 . 5 0}$ & $\mathbf{5 3 . 5 9}$ & $\mathbf{4 5 . 4 4}$ & $\mathbf{4 7 . 9 2}$ \\
$151-200$ & 24.99 & 23.69 & 28.60 & 25.21 \\
$201-250$ & 9.41 & 8.93 & 9.94 & 9.41 \\
$251-300$ & 4.02 & 2.52 & 1.83 & 3.66 \\
$>300$ & 2.66 & 1.17 & 3.04 & 2.54 \\
\hline
\end{tabular}

Table 2: Statistics of the question and answer lengths on our dataset.

Table 3: Statistics of the passage lengths on our dataset.

\subsection{Type-based analysis}

In this section, we analyze the Dev set in terms of different types such as question type, reasoning type, and answer type. Because Vietnamese is a subject-verb-object language similar to Chinese (Nguyen et al., 2018), Vietnamese question types in UIT-ViQuAD follow a manner in CMRC (Cui et al., 2019b). Thus, we also divide the questions into seven types: Who, What, When, Where, Why, How, and Others. However, in Vietnamese, question words vary a lot, so we have Workers manually annotate the type of questions. Figure $3 \mathrm{a}$ presents the distribution of the question types on our dataset. What questions account for the largest proportion of $49.97 \%$. Compared to SQuAD, the percentage of the What question in our dataset is similar to that in SQuAD (53.60\%) (Aniol et al., 2019).

To explore the difficulty of reasoning required, we conduct human annotation for the different reasoning level of the question, shown in Figure 3b. Following Hill et al. (2015) and Nguyen et al. (2020), workers manually annotate the questions into five different types of reasoning with ascending order of difficulty: word matching (WM), paraphrasing (PP), single-sentence reasoning (SSR), multisentence reasoning (MSR), and ambiguous/insufficient (AoI). Our dataset is more difficult than SQuAD and NewsQA because the percentage of inference types (68.29\%) in our dataset is higher than that in SQuAD (20.5\%) and NewsQA (33.90\%) (Trischler et al., 2017).

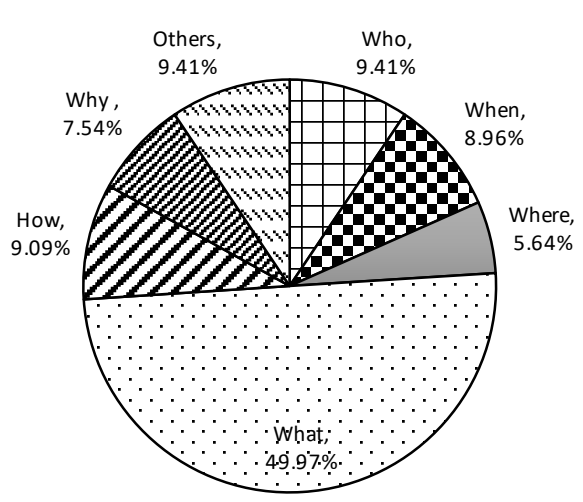

(a) Question Type

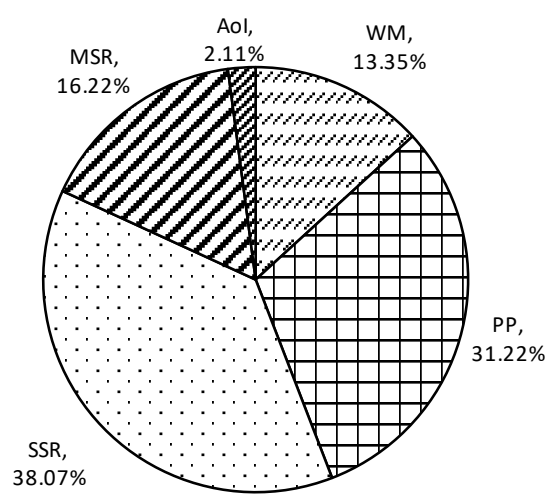

(b) Reasoning Type

Figure 3: The distribution of the question and reasoning types on the Dev set of UIT-ViQuAD.

Following Rajpurkar et al. (2016) and Trischler et al. (2015), we categorize answers based on their linguistic types such as time (N1), other numeric (N2), person (E1), location (E2), other entity (E3), noun phrase (P1), verb phrase (P2), adjective phrase (P3), preposition phrase (P4), clause (P5) and others (O). 
Unlike SQuAD (Rajpurkar et al., 2016) and NewsQA (Hill et al., 2015), instead of using automatic tools for annotation, the answer types on the Dev set of UIT-ViQuAD are annotated entirely by workers. Table 4 shows the distribution of the answer types based on various syntactic structures on the Dev set of our dataset. Common noun phrases account for the largest proportion in UIT-ViQuAD, which is similar to the statistics of SQuAD (Rajpurkar et al., 2016) and NewsQA (Trischler et al., 2017). In addition, verb phrases (P2) and other entities (E3) rank the second and third percentages in our dataset.

\begin{tabular}{cccccccccccc}
\hline Answer type & N1 & N2 & E1 & E2 & E3 & P1 & P2 & P3 & P4 & P5 & O \\
\hline Percentage & 7.71 & 9.41 & 5.39 & 4.32 & $\mathbf{1 1 . 6 5}$ & $\mathbf{2 2 . 8 6}$ & $\mathbf{1 8 . 4 3}$ & 2.52 & 3.18 & 5.91 & 10.55 \\
\hline
\end{tabular}

Table 4: Statistics of the answer types on the Dev set of the UIT-ViQuAD dataset.

\section{Empirical evaluation}

In this section, we conduct experiments with the state-of-the-art MRC models to evaluate our dataset. To measure the difficulty of our dataset, we also estimate human performance on the task of Vietnamese MRC. Similar to evaluations on English and Chinese datasets (Rajpurkar et al., 2016; Cui et al., 2019b), we used two evaluation metrics, exact match (EM) and F1-score, to evaluate performances of MRC models on our dataset.

\subsection{Human performance}

In order to measure human performance on the development and test sets, we hired three other workers to independently answer questions on the test and development sets. As a result, each question in the development ans test sets has four answers, as described in Phase 5 of Section 3 . Unlike Rajpurkar et al. (2016) and like Cui et al. (2019b), to measure the performance, we use a cross-validation methodology. In particular, we consider the first answer as human prediction and treat the remainder of the answers as ground truths. We obtain three human prediction performances by iteratively regarding the first, second, and third answer as the human prediction. We take the maximum performance over all of the ground truth answers for each question. Lastly, we calculate the average of four results as the final human performance on the dataset.

\subsection{Re-implemented methods and baselines}

In this paper, we re-implemented the following MRC models on our dataset as described in Section 4

- DrQA: Chen et al. (2017) introduced a simple but effective neural network-based model for the MRC task. DrQA Reader achieved good performance on multiple MRC datasets (Rajpurkar et al., 2016; Reddy et al., 2019; Labutov et al., 2018). Thus, we re-implement this method into our dataset as the first baseline models to compare future models.

- QANet: QANet was proposed by Yu et al. (2018) and this model also demonstrated good performance on multiple MRC datasets (Rajpurkar et al., 2016, Dua et al., 2019). This model consists of multiple convolutional layers followed by two components: the self-attention and fully connected layer, for both question and passage encoding as well as some more layers stacked before predicting the final output.

- BERT: BERT was proposed by Devlin et al. (2019). This model is a strong methodology for pre-training language representations, which achieved the state-of-the-art results on many reading comprehension tasks. In this paper, we used mBERT (Devlin et al., 2019), a large-scale multilingual language model pre-trained for the evaluation of our Vietnamese MRC task.

- XLM-R: XLM-R was proposed by Conneau et al. (2020), a super strong methodology for pretraining multilingual language models at scale, which leads to significant performance gains for a wide range of cross-lingual transfer tasks. This model significantly outperforms multilingual BERT (mBERT) on a variety of crosslingual benchmarks, including XNLI, MLQA, and NER. In this paper, we evaluate XLM-R $R_{\text {Base }}$ and XLM- $R_{\text {Large }}$ on our dataset. 


\subsection{Experimental settings}

We use a single NVIDIA Tesla P100 GPU via Google Colaboratory to train all MRC models on our dataset. We utilize the pre-trained word embeddings introduced by (Xuan et al., 2019), including

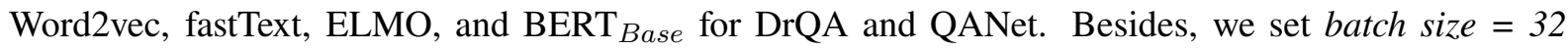
and epochs $=40$ for both the two models. To evaluate BERT on our dataset, we implement a multilingual pre-trained model mBERT (Devlin et al., 2019) and pre-trained cross-lingual models XLM-R (Conneau et al., 2020) with the baseline configuration provided by HuggingFace 3 , Based on our dataset characteristics, we use the maximum answer length to 300 , the question length to 64 , and the input sequence length to 384 for all the experiments on mBERT and XLM-R.

\begin{tabular}{|c|c|c|c|c|}
\hline \multirow{2}{*}{ Model } & \multicolumn{2}{|c|}{ EM } & \multicolumn{2}{|c|}{ F1-score } \\
\hline & Dev & Test & Dev & Test \\
\hline DrQA + Word2vec & 39.04 & 38.10 & 60.31 & 60.38 \\
\hline DrQA + FastText & 35.93 & 35.61 & 59.33 & 58.67 \\
\hline DrQA + ELMO & 43.98 & 40.91 & 65.09 & 63.44 \\
\hline DrQA + BERT & 35.71 & 34.84 & 58.00 & 57.73 \\
\hline QANet + Word2vec & 45.19 & 40.89 & 67.73 & 64.60 \\
\hline QANet + FastText & 39.66 & 46.05 & 63.82 & 68.06 \\
\hline QANet + ELMO & 46.10 & 42.21 & 67.62 & 65.76 \\
\hline QANet + BERT & 43.13 & 41.93 & 66.54 & 65.45 \\
\hline mBERT & 62.20 & 59.28 & 80.77 & 80.00 \\
\hline XLM-R Base & 63.87 & 63.00 & 81.90 & 81.95 \\
\hline XLM-R $\mathrm{R}_{\text {Large }}$ & 69.18 & 68.98 & 87.14 & 87.02 \\
\hline Human performance & 85.65 & 85.59 & 95.19 & 94.69 \\
\hline
\end{tabular}

Table 5: Human and model performances on the Dev and Test sets of UIT-ViQuAD.

\subsection{Evaluation results}

Table 5 presents the performance of our models alongside human performance on the development and test sets of our dataset. For EM and F1-core, XLM-R $\mathrm{L}_{\text {Large }}$ significantly outperforms the other models but is largely below human performance. On the test set, the model predicts answers with the F1-score of $87.02 \%$. However, this model's exact match achieves $68.98 \%$, which is significantly lower than the F1-score.

\subsection{Analysis}

To gain more in-depth insights into the evaluation of the machine models and humans in Vietnamese, we analyze their performances in terms of different linguistic aspects such as length-based (question length, answer length, and passage length) and type-based (question type, answer type, and reasoning type).

\subsubsection{Effects of length-based aspects}

In order to examine how well the MRC models could perform on UIT-ViQuAD, we analyze the performances of the machine models and humans by F1-score. Figure 4 shows length-based analyses of humans and MRC models' performances on the Dev set. In general, the performances of the mBERT and XLM-R models outperform that of the QANet and DrQA models. However, all machine models' performances are lower than humans on different types of lengths. For the question-length-based analysis (see Figure 4a), we found that longer questions tend to achieve better results because these questions maybe contain more information, which makes it easier for MRC models to find answers. On the contrary, the longer answers achieve lower performances, which is challenging for the MRC models, shown clearly in the performances of the DrQA and QANet models in Figure 4b. Unlike question-length and answer-length analyses, the passage lengths witness fluctuations in the performances of most MRC models work well

\footnotetext{
${ }^{3}$ https://huggingface.co
} 
for short ( $<100$ words) and long ( $>250$ words) passages (see Figure $4 \mathrm{c}$ ). The result analyses based on the different lengths can be used to evaluate the difficulty of Vietnamese automatic reading comprehension on our dataset, which can help researchers have ideas for curriculum learning in future work.

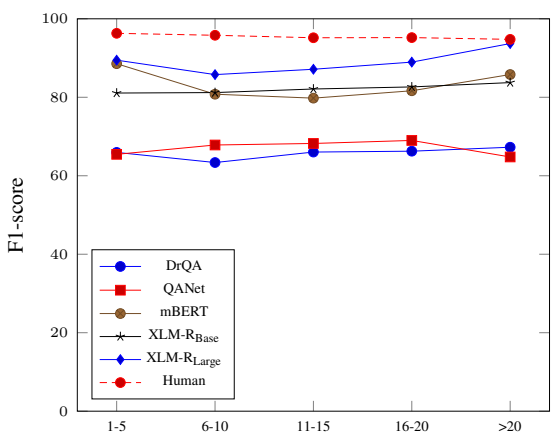

(a) Question length.

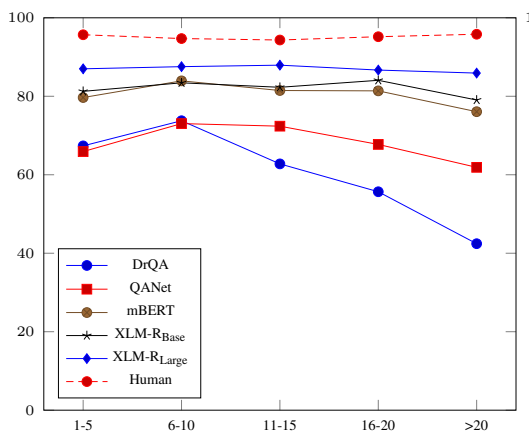

(b) Answer length.

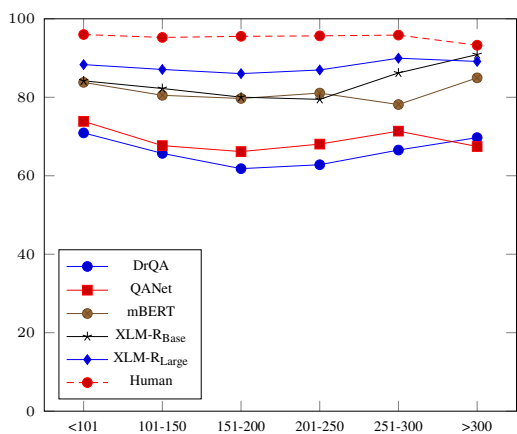

(c) Passage length.

Figure 4: Length-based analysis of F1-score performances on the Dev set of UIT-ViQuAD.

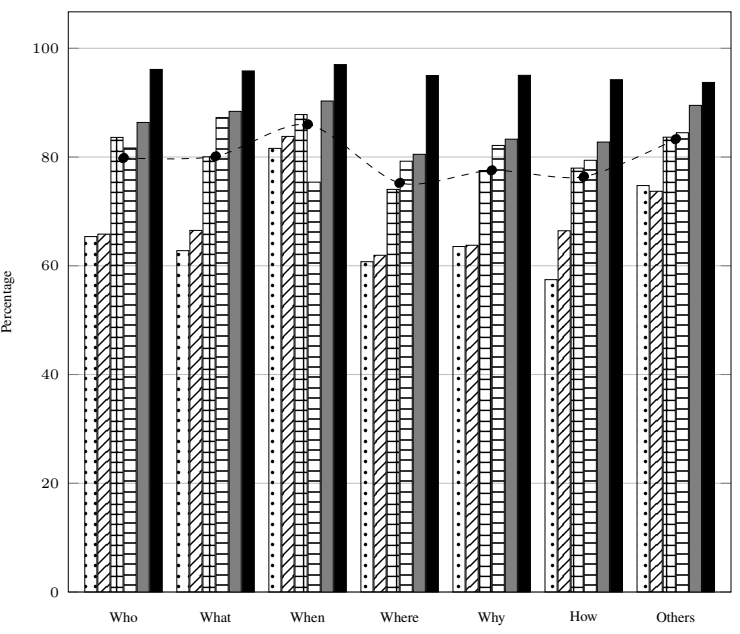

(a) Question type.

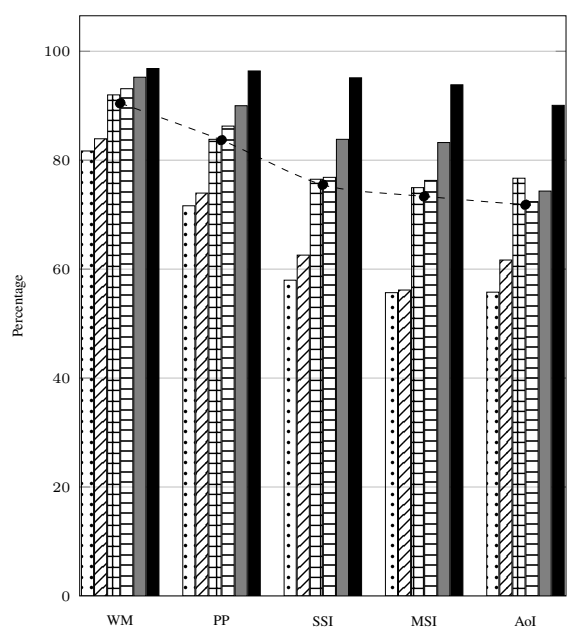

(b) Reasoning type.
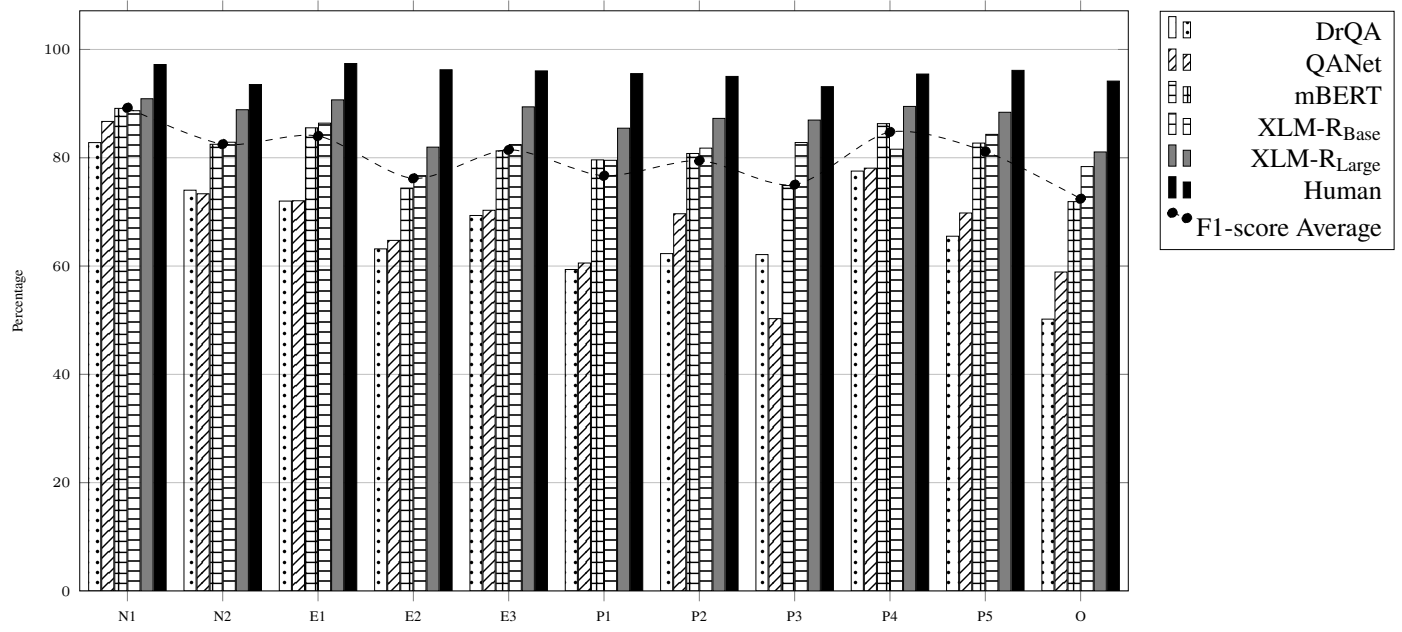

(c) Answer type.

Figure 5: Type-based analysis of F1-score performances on the Dev set of UIT-ViQuAD. The lines on the graphs are the average of F1-score performances on the MRC models. 


\subsubsection{Effects of type-based aspects}

Besides, we examine how MRC models solve the type-based aspects of UIT-ViQuAD. Therefore, we analyze the F1-score performances of the machine models and humans on the development. Figure 5 shows the type-based analyses of humans and MRC models' performances. No machine models have been able to handle question types, answer types, and types of reasoning better than humans. On the type of reasoning, complex inference types (SSI, MSI, and AoI) obtain lower performances, which is similar to results on SQuAD and NewsQA (Trischler et al., 2017). Similarly, difficult question types (Why and How) obtain low performances. However, the Where question is also another question type that does not been handle well in machine models. Thus, the Location answer type related to the Where question type also achieves low performances. Although the noun-phrase answer type accounts for the highest proportion of the dataset $(22.86 \%)$, the machine model does not yet handle well as other types because of the diverse and complicated structure of Vietnamese noun phrases (Nguyen et al., 2018).

\subsubsection{Effects of the amount of training data}

The training data consists of 18,579 question-answer pairs which are lower than the quantity of the data trained for English and Chinese MRC models. To verify whether the small amount of training data affect the poor performance of the MRC systems based on model evaluations, we conduct various experiments with training sets comprising 3,145, 6,471, 9,268, 12,273, 15,145, and 18,579 questions. Figure 6 shows the performance (F1-score) based on the Test set of UIT-ViQuAD. Through these experimental analyses, we find that DrQA, QANet, and mBERT obtain better performances when the amount of training data increases, whereas the performances of XLM-R are stable over $86 \%$ with any training data amount. These observations indicate that the best model $\left(\mathrm{XLM}-\mathrm{R}_{\text {Large }}\right)$ is more effective with a small amount of training data compared with the other three models. In general, increasing the training data quantity may be required to improve the performance of future models for most of neural network-based MRC models.

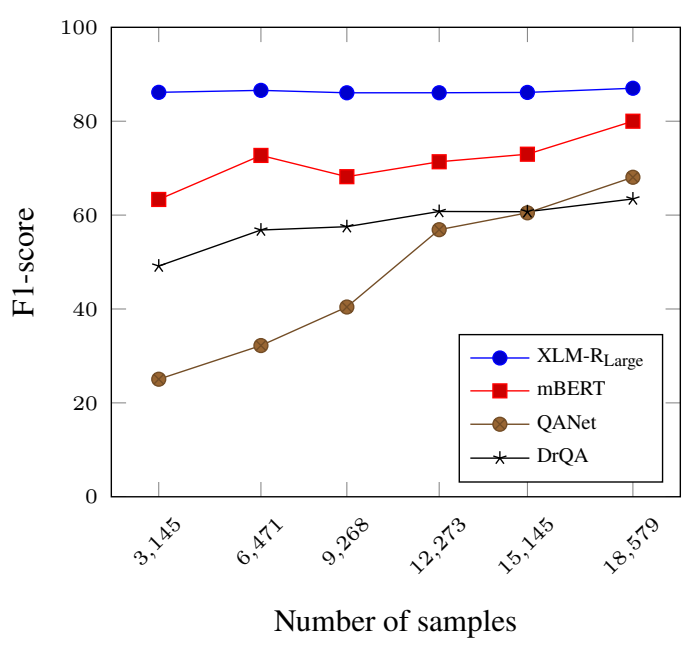

Figure 6: The impact of the amount of training data on the Test set of UIT-ViQuAD.

\section{Conclusion and future work}

In this paper, we introduce a new span-extraction dataset for evaluating Vietnamese MRC. UIT-ViQuAD contains over 23,000 questions generated by humans. Our experimental results show that the machines could obtain up to 87 percent scores on both the development and test set. However, they are lower than the estimated human performances in F1-score. We hope the release of our dataset contributes to the language diversity in MRC task, and accelerates further investigation on solving difficult questions that need comprehensive reasoning over multiple clues. According to the analysis results, we may extend this work by exploring models to solve challenging questions involving specific question types (Where, Why, and How), answer types (Location, and Noun Phrases) and reasoning types (Single-Sentence Inference, Multiple-Sentence Inference, Ambiguous or Insufficient). In future, we plan to enhance the quantity and 
the quality of our dataset to achieve better performance on deep learning and transformer models. In addition, we would like to open the Vietnamese MRC challenging task for researchers in the field.

\section{Acknowledgements}

We would like to thank the reviewers' comments which are helpful for improving the quality of our work. In addition, we would like to thank our workers for their cooperation.

\section{References}

Anna Aniol, Marcin Pietron, and Jerzy Duda. 2019. Ensemble Approach for Natural Language Question Answering Problem. In Seventh International Symposium on Computing and Networking Workshops (CANDARW), pages 180-183. IEEE.

Danqi Chen, Adam Fisch, Jason Weston, and Antoine Bordes. 2017. Reading Wikipedia to Answer Open-Domain Questions. In Proceedings of the 55th Annual Meeting of the Association for Computational Linguistics (Volume 1: Long Papers), pages 1870-1879.

Alexis Conneau, Kartikay Khandelwal, Naman Goyal, Vishrav Chaudhary, Guillaume Wenzek, Francisco Guzmán, Edouard Grave, Myle Ott, Luke Zettlemoyer, and Veselin Stoyanov. 2020. Unsupervised Cross-lingual Representation Learning at Scale. In Proceedings of the 58th Annual Meeting of the Association for Computational Linguistics, pages 8440-8451. Association for Computational Linguistics.

Yiming Cui, Ting Liu, Zhipeng Chen, Shijin Wang, and Guoping Hu. 2016. Consensus Attention-based Neural Networks for Chinese Reading Comprehension. In Proceedings of COLING 2016, the 26th International Conference on Computational Linguistics: Technical Papers, pages 1777-1786.

Yiming Cui, Wanxiang Che, Ting Liu, Bing Qin, Shijin Wang, and Guoping Hu. 2019a. Cross-Lingual Machine Reading Comprehension. In Proceedings of the 2019 Conference on Empirical Methods in Natural Language Processing and the 9th International Joint Conference on Natural Language Processing (EMNLP-IJCNLP), pages $1586-1595$.

Yiming Cui, Ting Liu, Wanxiang Che, Li Xiao, Zhipeng Chen, Wentao Ma, Shijin Wang, and Guoping Hu. 2019b. A Span-Extraction Dataset for Chinese Machine Reading Comprehension. In Proceedings of the 2019 Conference on Empirical Methods in Natural Language Processing and the 9th International Joint Conference on Natural Language Processing (EMNLP-IJCNLP), pages 5886-5891.

Jacob Devlin, Ming-Wei Chang, Kenton Lee, and Kristina Toutanova. 2019. BERT: Pre-training of Deep Bidirectional Transformers for Language Understanding. In Proceedings of the 2019 Conference of the North American Chapter of the Association for Computational Linguistics: Human Language Technologies, Volume 1 (Long and Short Papers), pages 4171-4186.

Dheeru Dua, Yizhong Wang, Pradeep Dasigi, Gabriel Stanovsky, Sameer Singh, and Matt Gardner. 2019. DROP: A Reading Comprehension Benchmark Requiring Discrete Reasoning Over Paragraphs. In Proceedings of the 2019 Conference of the North American Chapter of the Association for Computational Linguistics: Human Language Technologies, Volume 1 (Long and Short Papers), pages 2368-2378.

Xingyi Duan, Baoxin Wang, Ziyue Wang, Wentao Ma, Yiming Cui, Dayong Wu, Shijin Wang, Ting Liu, Tianxiang Huo, Zhen Hu, et al. 2019. CJRC: A Reliable Human-Annotated Benchmark DataSet for Chinese Judicial Reading Comprehension. In China National Conference on Chinese Computational Linguistics, pages 439451. Springer.

Wei He, Kai Liu, Jing Liu, Yajuan Lyu, Shiqi Zhao, Xinyan Xiao, Yuan Liu, Yizhong Wang, Hua Wu, Qiaoqiao She, et al. 2018. DuReader: a Chinese Machine Reading Comprehension Dataset from Real-world Applications. In Proceedings of the Workshop on Machine Reading for Question Answering, pages 37-46.

Karl Moritz Hermann, Tomas Kocisky, Edward Grefenstette, Lasse Espeholt, Will Kay, Mustafa Suleyman, and Phil Blunsom. 2015. Teaching machines to read and comprehend. In Advances in neural information processing systems, pages 1693-1701.

Felix Hill, Antoine Bordes, Sumit Chopra, and Jason Weston. 2015. The Goldilocks Principle: Reading Children's Books with Explicit Memory Representations. In International Conference on Learning Representations (ICLR). 
Hsin-Yuan Huang, Chenguang Zhu, Yelong Shen, and Weizhu Chen. 2018. FusionNet: Fusing via Fully-aware Attention with Application to Machine Comprehension. In International Conference on Learning Representations.

Igor Labutov, Bishan Yang, Anusha Prakash, and Amos Azaria. 2018. Multi-Relational Question Answering from Narratives: Machine Reading and Reasoning in Simulated Worlds. In Proceedings of the 56th Annual Meeting of the Association for Computational Linguistics (Volume 1: Long Papers), pages 833-844.

Guokun Lai, Qizhe Xie, Hanxiao Liu, Yiming Yang, and Eduard Hovy. 2017. RACE: Large-scale ReAding Comprehension Dataset From Examinations. In Proceedings of the 2017 Conference on Empirical Methods in Natural Language Processing, pages 785-794.

Seungyoung Lim, Myungji Kim, and Jooyoul Lee. 2019. KorQuAD1.0: Korean QA Dataset for Machine Reading Comprehension. arXiv preprint arXiv:1909.07005.

Quy T Nguyen, Yusuke Miyao, Ha TT Le, and Nhung TH Nguyen. 2018. Ensuring annotation consistency and accuracy for Vietnamese treebank. Language Resources and Evaluation, 52(1):269-315.

Kiet Van Nguyen, Khiem Vinh Tran, Son T. Luu, Anh Gia-Tuan Nguyen, and Ngan Luu-Thuy Nguyen. 2020. Enhancing lexical-based approach with external knowledge for Vietnamese multiple-choice machine reading comprehension. arXiv preprint arXiv:2001.05687.

Pranav Rajpurkar, Jian Zhang, Konstantin Lopyrev, and Percy Liang. 2016. SQuAD: 100,000+ Questions for Machine Comprehension of Text. In Proceedings of the 2016 Conference on Empirical Methods in Natural Language Processing, pages 2383-2392.

Pranav Rajpurkar, Robin Jia, and Percy Liang. 2018. Know What You Don't Know: Unanswerable Questions for SQuAD. In Proceedings of the 56th Annual Meeting of the Association for Computational Linguistics (Volume 2: Short Papers), pages 784-789.

Siva Reddy, Danqi Chen, and Christopher D Manning. 2019. CoQA: A Conversational Question Answering Challenge. Transactions of the Association for Computational Linguistics, 7:249-266.

Matthew Richardson, Christopher JC Burges, and Erin Renshaw. 2013. MCTest: A Challenge Dataset for the Open-Domain Machine Comprehension of Text. In Proceedings of the 2013 Conference on Empirical Methods in Natural Language Processing, pages 193-203.

Minjoon Seo, Aniruddha Kembhavi, Ali Farhadi, and Hannaneh Hajishirzi. 2017. Bidirectional Attention Flow for Machine Comprehension. In International Conference on Learning Representations (ICLR).

Chih Chieh Shao, Trois Liu, Yuting Lai, Yiying Tseng, and Sam Tsai. 2018. DRCD: a Chinese Machine Reading Comprehension Dataset. arXiv preprint arXiv:1806.00920.

Kai Sun, Dian Yu, Jianshu Chen, Dong Yu, Yejin Choi, and Claire Cardie. 2019. DREAM: A Challenge Data Set and Models for Dialogue-Based Reading Comprehension. Transactions of the Association for Computational Linguistics, 7:217-231.

Adam Trischler, Tong Wang, Xingdi Yuan, Justin Harris, Alessandro Sordoni, Philip Bachman, and Kaheer Suleman. 2017. NewsQA: A Machine Comprehension Dataset. In Proceedings of the 2nd Workshop on Representation Learning for NLP, pages 191-200.

Shuohang Wang and Jing Jiang. 2016. Machine Comprehension Using Match-LSTM and Answer Pointer. arXiv preprint arXiv:1608.07905.

Wenhui Wang, Nan Yang, Furu Wei, Baobao Chang, and Ming Zhou. 2017. Gated Self-Matching Networks for Reading Comprehension and Question Answering. In Proceedings of the 55th Annual Meeting of the Association for Computational Linguistics (Volume 1: Long Papers), pages 189-198.

Dirk Weissenborn, Georg Wiese, and Laura Seiffe. 2017. Making Neural QA as Simple as Possible but not Simpler. In Proceedings of the 21st Conference on Computational Natural Language Learning (CoNLL 2017), pages 271-280.

Son Vu Xuan, Thanh Vu, Son Tran, and Lili Jiang. 2019. ETNLP: A Visual-Aided Systematic Approach to Select Pre-Trained Embeddings for a Downstream Task. In Proceedings of the International Conference on Recent Advances in Natural Language Processing (RANLP 2019), pages 1285-1294.

Adams Wei Yu, David Dohan, Minh-Thang Luong, Rui Zhao, Kai Chen, Mohammad Norouzi, and Quoc V Le. 2018. QANet: Combining Local Convolution with Global Self-Attention for Reading Comprehension. In International Conference on Learning Representations. 